\title{
Galenus-Preis: Nivolumab verstärkt die Immunantwort
}

— In der Behandlung von Patienten mit fortgeschrittenen Krebserkrankungen sind Immun-Checkpoint-Inhibitoren inzwischen etablierte Therapieoptionen. CheckpointInhibitoren bekämpfen die Tumorzellen nicht direkt, sondern verstärken und aktivieren die gegen den Tumor gerichtete körpereigene Immunabwehr darin, gegen Tumorzellen vorzugehen. $\mathrm{Zu}$ diesen Checkpoint-Inhibitoren gehört Nivolumab $\left(\mathrm{Opdivo}^{\circledR}\right)$ von Bristol-Myers Squibb. Nivolumab ist der erste Vertreter der neuen Wirkstoffklasse der PD-1(„programmed death 1")-Inhibitoren und wurde im Juni 2015 in der EU zur Monotherapie von erwachsenen Patienten mit fortgeschrittenem (nicht resezierbarem oder metastasiertem) Melanom zugelassen und in Deutschland auf den Markt gebracht. Im Mai 2016 folgte EU-weit die erste Zulassung von $\mathrm{Ni}$ - volumab in Kombination mit Ipilimumab (Yervoy ${ }^{\oplus}$ ), ebenfalls einem Immun-Checkpoint-Inhibitor, für diese Indikation.

Das Prinzip der Immun-Checkpoint-Inhibition hat sich zu einem zentralen Hoffnungsträger für viele Indikationsbereiche der Krebstherapie entwickelt. Denn die therapieinduzierte Verstärkung der Immunreaktion führte bei einem relevanten Anteil von Tumorpatienten im Vergleich zur bisherigen Standardtherapie zu einem deutlichen Überlebensvorteil. So profitierten Patienten mit fortgeschrittenem Melanom vom BRAF-Wildtyp in der Phase-III-Studie CheckMate 066 unter der Erstlinientherapie mit Nivolumab von einer signifikanten Verlängerung des Gesamtüberlebens. Dieses lag nach einem Jahr bei 72,9\% versus $42,1 \%$ unter Chemotherapie mit Dacarbazin. Im zweiten Jahr fiel die Gesamtüberlebensrate unter Nivolumab versus Da- carbazin mehr als doppelt so hoch aus $(57,7 \%$ vs. $26,7 \%$; $p<0,001$ ).

Die synergistische Wirkung der Kombination von Nivolumab mit Ipilimumab bei nicht vorbehandelten Patienten mit fortgeschrittenem Melanom wurde in der Phase-IIIStudie CheckMate 067 deutlich: Das mediane progressionsfreie Überleben nach 18 Monaten betrug mit der Kombinationstherapie 11,5 Monate, im Nivolumab-Arm 6,9 Monate ( je p , 0,001 vs. Ipilimumab) und im Ipilimumab-Arm 2,9 Monate).

Nivolumab ist zudem zur Behandlung von Patienten mit lokal fortgeschrittenem oder metastasiertem nicht kleinzelligem Lungenkarzinom (NSCLC) mit und ohne Plattenepithelhistologie nach vorheriger Chemotherapie zugelassen sowie zur Monotherapie bei Patienten mit fortgeschrittenem Nierenzellkarzinom nach Vortherapie. Yuri Sankawa

\section{Galenus-Preis: Pembrolizumab mit attestiertem Zusatznutzen bei fortgeschrittenem Melanom}

— Pembrolizumab (Keytruda ${ }^{\circledR}$ ) ist ein monoklonaler Antikörper, der an den PD1(„programmed death 1“)-Rezeptor auf den T-Zellen bindet und als Checkpoint-Inhibitor wirkt. Das Präparat von MSD Sharp \& Dohme ist seit August 2015 in Deutschland als Monotherapie zur Behandlung des fortgeschrittenen, nicht resezierbaren oder meta-

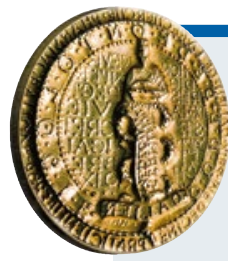

Galenus-von-PergamonPreis

Der Galenus-von-PergamonPreis würdigt herausragende Arzneimittel-Innovationen und exzellente Forschungsleistung in der klinischen oder experimentellen Pharmakologie. In diesem Jahr ist er für die drei Kategorien Primary Care, Specialist Care und Orphan Drugs ausgeschrieben worden. Der Galenus-von-PergamonPreis 2016 wird am 20. Oktober 2016 in Berlin verliehen. In diesem Jahr befinden sich mit Keytruda ${ }^{\circledR}$ und Opdivo ${ }^{\circledR}$ zwei Medikamente aus der Dermatologie unter den Bewerbern. stasierten Melanoms bei Erwachsenen verfügbar.

In der Phase-II-Studie KEYNOTE-002 erhielten $\mathbf{5 4 0}$ mit Ipilimumab und zum Teil mit einem BRAF- oder MEK-Inhibitor vorbehandelte Patienten mit fortgeschrittenem Melanom randomisiert entweder Pembrolizumab in Dosierungen von $2 \mathrm{mg} / \mathrm{kg}$ Körpergewicht oder $10 \mathrm{mg} / \mathrm{kg} \mathrm{KG}$ alle drei Wochen oder Chemotherapie. Nach sechs Monaten betrug das progressionsfreie Überleben (PFS) mit 2 mg/kg KG Pembrolizumab 34\%, mit 10 mg/ kg KG Pembrolizumab 38\% und mit Chemotherapie $16 \%$. Die Gesamtansprechraten lagen bei $21 \%$ und $25 \%$ mit Pembrolizumab und waren damit signifikant höher als bei Chemotherapie ( $4 \%$; je $p<0,0001)$. Therapiebedingte Nebenwirkungen vom Grad 3-4 waren unter Pembrolizumab mit $11 \%$ (2 mg/ $\mathrm{kg} \mathrm{KG})$ und $14 \%$ (10 mg/kg KG) seltener als unter Chemotherapie (26\%).

In der Phase-III-Studie KEYNOTE-006 wurde die Pembrolizumab-Therapie bei 834 Patienten mit fortgeschrittenem Melanom im Stadium III oder IV, die zuvor nicht mehr als eine systemische Therapie erhalten hatten, direkt mit einer Ipilimumab-Therapie verglichen. Randomisiert erhielten die Studienteilneh- mer Pembrolizumab 10 mg/kg KG alle zwei oder drei Wochen im Vergleich zu vier Zyklen Ipilimumab $3 \mathrm{mg} / \mathrm{kg} \mathrm{KG}$ alle drei Wochen. Bei der ersten vordefinierten Interimsanalyse betrug das geschätzte Sechs-Monats-PFS mit Pembrolizumab alle zwei Wochen 47,3\%, mit Pembrolizumab alle drei Wochen $46,4 \%$ und mit Ipilimumab 26,5\%. Das Ein-JahresGesamtüberleben lag für die Patienten mit Pembrolizumab alle zwei Wochen bei $74,1 \%$, mit Pembrolizumab alle drei Wochen bei $68,4 \%$ und mit Ipilimumab bei $58,2 \%$.

Aufgrund der Studiendaten hat der G-BA im Februar 2016 Pembrolizumab einen beträchtlichen Zusatznutzen zugesprochen. Bei vorbehandelten Patienten mit fortgeschrittenem Melanom, für die Ipilimumab als nächste Therapie geeignet ist, treten bei numerisch längerem Gesamtüberleben schwere unerwünschte Ereignisse sowie Therapieabbrüche wegen unerwünschter Ereignisse unter Pembrolizumab später auf. Nicht vorbehandelte Erwachsene ohne BRAF-V600-Mutation überleben mit dem neuen Wirkstoff länger und profitieren von der verbesserten Verträglichkeit und Lebensqualität. Auch hier wurde ein beträchtlicher Zusatznutzen zuerkannt. Susanne Heinzl 